\title{
Design of an Electron Gun for an 8 MW Peak, 30kW Average Power S-Band Klystron
}

\author{
Deepender Kant* and Atmakuru Nagaraju \\ CSIR-Central Electronics Engineering Research Institute, Pilani, India \\ *Corresponding author's email: dkc@ceeri.res.in
}

\begin{abstract}
High Power S-band pulsed klystrons with peak power greater than $6 \mathrm{MW}$ are widely used as RF sources in particle accelerators. This paper presents an electron gun (pierce type) design for such a klystron with an output power of 8 MW peak and $30 \mathrm{~kW}$ average with its intended applications in accelerators for food processing. The targeted device is currently under design at CSIR-CEERI and will be developed later on. The results of electron gun simulation through computational codes like Trak, CST particle studio, and Opera are compared to find an optimum design for the electron gun to be fabricated for the klystron.
\end{abstract}

Keywords: Power Klystron, Electron gun, TRAK, CST, Opera

\section{Introduction}

High power klystron plays a vital part in many applications, including particle accelerator, collider, food processing unit, synchrotron radiation source, and freeelectron laser. As performance requirement for these applications is getting increasingly challenging, klystron technology must also keep pace to afford the essential high power and efficiency in these applications [1], [2].

In recent years, microwaves in food processing are increasingly popular with commercial cooking, drying, tempering, sterilization, etc. The main advantages of this technique are low processing time, increased safety, reducing spoilage, a cleaner work environment, and high nutrient retention compared to conventional heating [3]. In this paper, the design of an electron-gun is proposed for its use in S-band klystron with peak power of $8 \mathrm{MW}$ and $30 \mathrm{~kW}$ average power, which is intended for its end use as RF source in a particle accelerator system being developed for food processing applications. Initial dimensions of the electron gun are taken from the Vaughan synthesis [4], initial optimization of dimensional parameters have been done with Trak software [5], and final dimensions of the electron gun with and without magnetic field are validated with CST Particle studio [6] and Opera-3D [7] codes.

\section{Design of Electron gun}

The design of an electron gun is an essential and primary step towards creating a klystron, as optimization of the beam optics is essentially needed before any RF interaction mechanism. The primary role of the electron gun in a klystron is to provide an electron beam of desired characteristics for interaction with the RF fields. It consists of a spherical cathode with the emitting surface formed of some low work function material. The electrons emitted by the cathode through the thermionic mechanism are accelerated by a concentric anode kept at a positive DC potential with respect to the cathode. The converging electron beam is given a laminar cylindrical shape with the help of a beam-focusing electrode (BFE) that is usually kept at cathode potential and the diverging lens formed by suitably shaped anode with an aperture at the center for passage of electron beam. The shape of the beam is maintained with the help of a coaxial magnetic field with flux lines parallel to axis. The schematic diagram of such a gun is shown in Figure 1, which is known to be a Pierce-type design [4].

Considering the target efficiency around $45 \%$ and produce the peak output power of $8 \mathrm{MW}$, the electron beam parameters of the electron gun are taken as $150 \mathrm{kV}$ beam voltage and 120 A beam current. Here targeted beam radius is $9.8 \mathrm{~mm}$, which has been derived by considering other parameters such as operational frequency, beam fill factor, etc. [8-10]. The magnetic focusing circuit is designed such that a small fraction of the magnetic field needed for focusing links at the cathode with flux lines parallel to the electron trajectories. The initial estimation of the shape of the electrodes has been done using the synthesis approach of Vaughan. A computer program has been written that generates the basic dimensional information about the electrodes for a beam of desired characteristics at rated beam voltage and current, essentially solving the electrostatic Poisson's equation with given boundary conditions. The input parameters used for the program are given in Table 1, and the computed output parameters are listed in Table 2, which are used for modeling of the electron gun structure in various simulation codes.

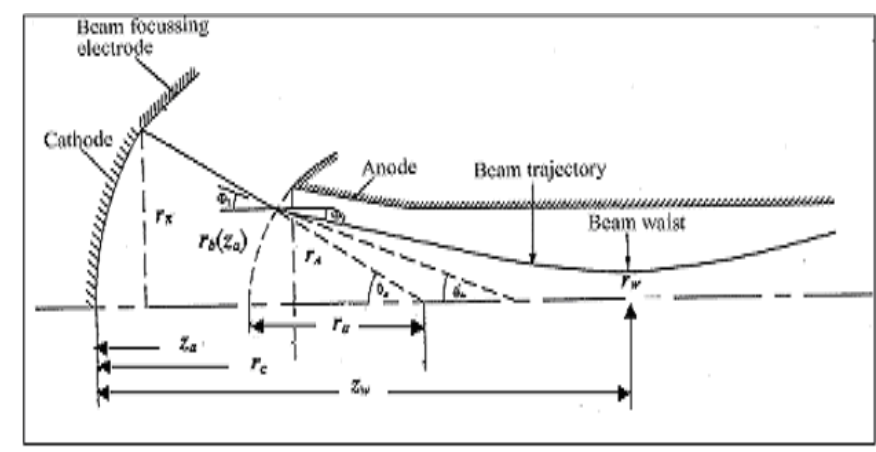

Fig1. Line diagram of Pierce electron gun showing the geometrical parameters used in the analysis 
Table 1. Input design parameters

\begin{tabular}{|l|l|}
\hline Parameters & Value \\
\hline Beam voltage $\left(\mathrm{V}_{0}\right)$ & $150 \mathrm{kV}$ \\
\hline Beam current $\left(\mathrm{I}_{0}\right)$ & $120 \mathrm{~A}$ \\
\hline Beam waist radius & $9.8 \mathrm{~mm}$ \\
\hline Emission density & $5 \mathrm{~A} / \mathrm{cm}^{2}$ \\
\hline
\end{tabular}

Table 2. Computed design parameters

\begin{tabular}{|l|c|}
\hline Design parameters & Computed value \\
\hline Beam perveance & $2.06 \mu \mathrm{P}$ \\
\hline Convergence half angle & $28.565176 \mathrm{Deg}$. \\
\hline Cathode disk radius & $27.64 \mathrm{~mm}$ \\
\hline Cathode spherical radius & $\sim 57.8 \mathrm{~mm}$ \\
\hline Anode spherical radius & $27.7 \mathrm{~mm}$ \\
\hline Beam radius at anode plane & $13.2 \mathrm{~mm}$ \\
\hline Radii of anode aperture & $15.9 \mathrm{~mm}$ \\
\hline Anode cathode spherical spacing & $30.1 \mathrm{~mm}$ \\
\hline Anode cathode axial spacing & $35.1 \mathrm{~mm}$ \\
\hline Throw from cathode vertex to anode & $77.7 \mathrm{~mm}$ \\
\hline
\end{tabular}

For this purpose, TRAK is used and the electrode shapes optimized after several runs iteratively. The results at the end of each run are scrutinized and necessary geometric corrections are incorporated in the input file to affect the desired beam parameters. This procedure is followed iteratively till the desired electron beam parameters are obtained. The final optimized beam trajectories (without applying magnetic field) from TRAK code are shown in Figure 2. The computed beam current is $120 \mathrm{~A}$ at $150 \mathrm{kV}$ beam voltage. The throw $\left(\mathrm{Z}_{\mathrm{w}}\right)$ and beam waist radius $\left(\mathrm{r}_{\mathrm{w}}\right)$ are 78.0 $\mathrm{mm}$ and $9.8 \mathrm{~mm}$, respectively.

\subsection{Design of axial magnetic field using solenoid}

The shape of the electrodes is optimized to get the beam waist radius close to the final beam radius necessary for optimum interaction in the RF circuit. If the beam is allowed to drift beyond the point of the waist, it starts diverging due to space charge forces and follows the natural beam spread curve [8]. To maintain the minimum beam radius, apply an appropriate focusing magnetic field, restricting the beam divergence and allowing the beam to drift axially with a more or less constant radius.

The confined flow-focusing scheme [8] has been used for designing axial magnetic field in the present electron gun where Brillouin magnetic field $(\mathrm{Bb})$ is given by the following relation,

$$
B_{b}^{2}=69 \times\left(I / V_{b}^{0.5}\right) \times R_{b}^{2}
$$

where $\mathrm{I}=$ beam current, $\mathrm{R}_{\mathrm{b}}=$ beam radius and $\mathrm{V}_{\mathrm{b}}=$ beam voltage.

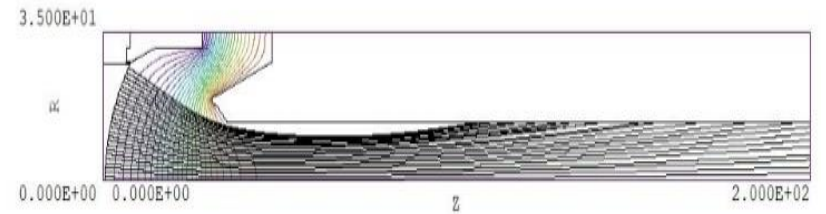

Fig 2. View of Electron Trajectories after simulation using TRAK
The computed value for $B_{b}$ is found as 472 Gauss. The required value of total axial magnetic field $\left(B_{t}\right)$ for confined flow-focusing will be about 2.5 times this value i.e., 1200 Gauss.

A solenoid with suitably shaped pole pieces is designed to achieve the desired magnetic field value for focusing of the beam. The borehole diameter of the pole piece decides the value of magnetic flux linked at the cathode surface $\left(B_{c}\right)$, which can be computed by Busch's theorem [8].

$$
B_{t}^{2}=B_{b}^{2}+B_{c}^{2} \times\left({ }^{A_{c}} / A_{e}\right)^{2}
$$

where $A_{c} / A_{e}$ is the area compression ratio of the electron gun.

The above ratio can be calculated by knowing the cathode surface area and beam cross-section area, this ratio is 8.4 in our case, so the computed value of cathode linkage magnetic flux is 131 Gauss. The borehole diameter is optimized at 100 $\mathrm{mm}$ so as to link the magnetic field value of about 130 Gauss near the cathode surface. The magnetic field profile after simulation of the solenoid is shown in Figure 3, where the magnetic field in the focusing region is around 1200 Gauss thereafter in the RF interaction region. The magnetic field profile is designed increasingly due to bunching occurring there.

Electron beam focused in the presence of axial magnetic field is shown in Figure 4, in Trak code, where minor scalloping of the beam is observed but without any crossover of electron trajectories. A close view of the trajectories indicates that the electron beam is propagating without any interception.

\section{Validation of Electron gun design}

The design parameters obtained from TRAK code are validated from the simulation study carried out using CST Particle studio and Opera-3D codes. Simulation of electron gun design depends on accurate modeling of potential lines near the emitting surface. For this reason, fine meshing is considered near the cathode, as shown in Figure 5.

In these computational codes, space charge emission is used and cathode as well as BFE are kept at ground potential while the anode is kept at $150 \mathrm{kV}$. The equipotential lines and electron trajectories of the simulated electron beam in CST Particle studio and OPERA-3D are shown in Figure 6 and 7, respectively.

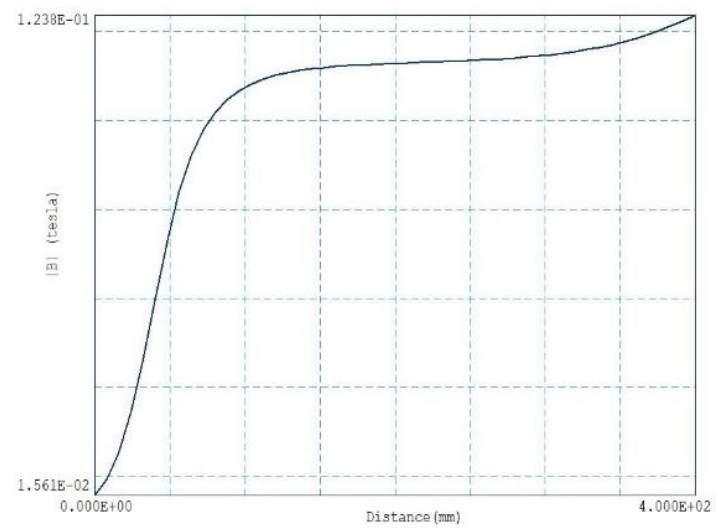

Fig 3. Magnetic Field Profile in Electron gun region 


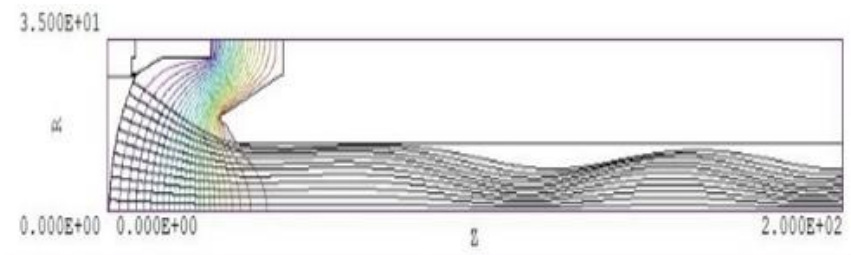

Fig 4. View of Electron Trajectories after applying magnetic field

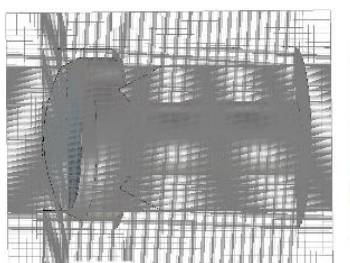

(a)

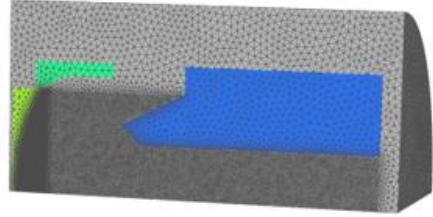

(b)
Fig. 5. Mesh for simulation. (a) CST Particle Studio. (b) OPERA-3D

To validate the magnet design of the klystron, the solenoid with suitable pole pieces is modeled in OPERA-3D software and the gun is simulated in the presence of a magnetic field and with its electron trajectories in the presence of magnetic field is shown in Figure 8.

Beam current and beam voltage characteristics of the simulated electron gun are plotted as shown in Figure 9 for a comparison of results through CST particle studio, Opera-3D, and Trak codes. These results are also shown in Table 3.

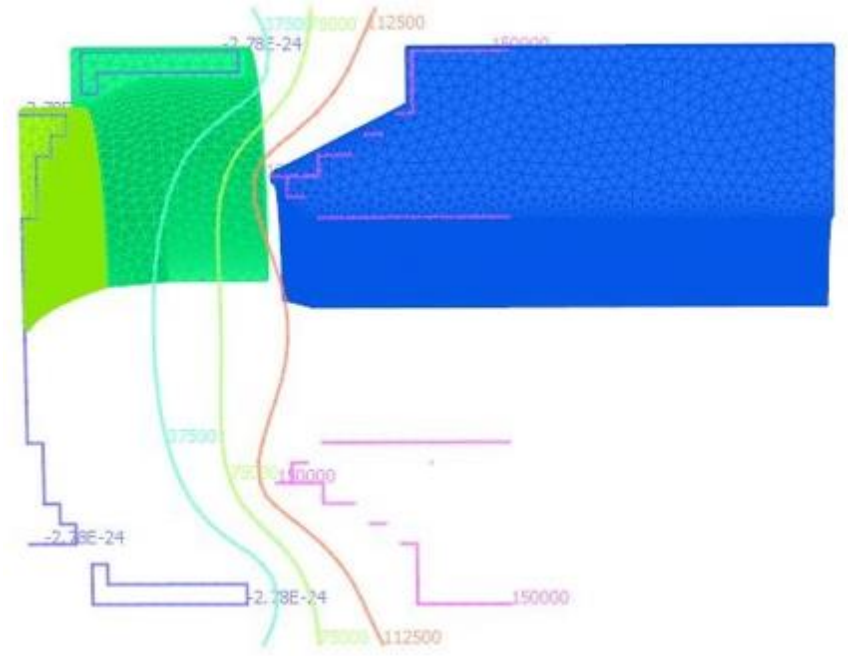

(a)

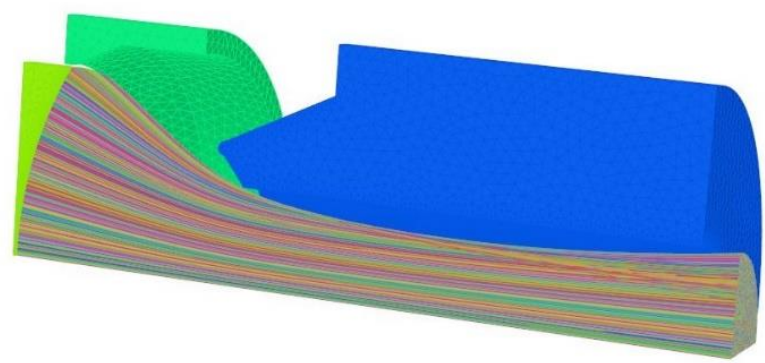

(b)

Fig. 6. Equipotential lines and beam trajectories in OPERA-3D. (a) Equipotential lines (b) beam trajectory

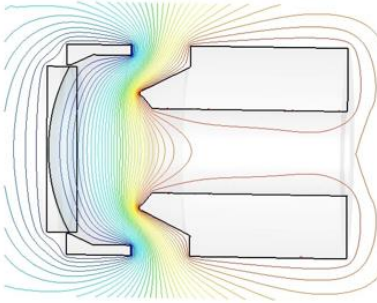

(a)

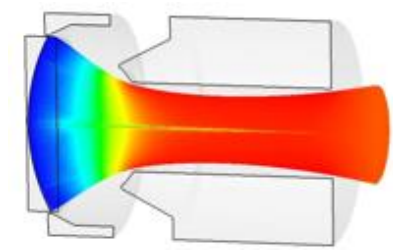

(b)
Fig. 7. Equipotential lines and beam trajectories in CST Particle studio. (a) Equipotential lines (b) beam trajectory.

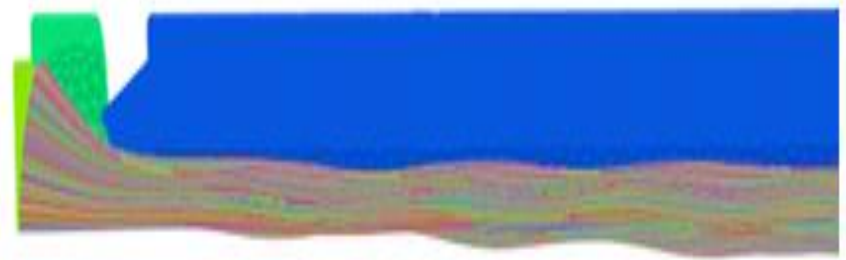

Fig. 8. Electron trajectory in the presence of a magnetic field in OPERA-3D.

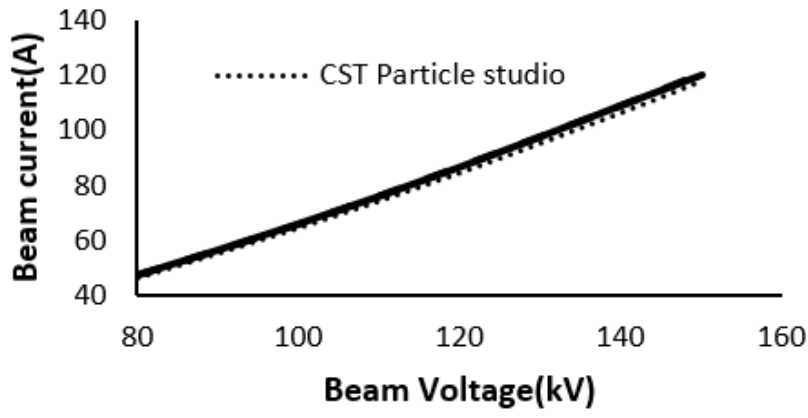

Fig. 9. Comparison of beam current with respect to beam voltage.

Table 3. Comparison of results with different codes

\begin{tabular}{|l|c|c|c|}
\hline $\begin{array}{l}\text { Beam } \\
\text { Voltage(kV) }\end{array}$ & $\begin{array}{l}\text { Beam } \\
\text { current(A) } \\
\text { (Trak) }\end{array}$ & $\begin{array}{l}\text { Beam } \\
\text { current(A) } \\
\text { (CST) }\end{array}$ & $\begin{array}{l}\text { Beam } \\
\text { current(A) } \\
\text { (Opera) }\end{array}$ \\
\hline 80 & 47.4 & 46.3 & 47.6 \\
\hline 100 & 66 & 64.5 & 66.0 \\
\hline 120 & 86.6 & 84.6 & 86.8 \\
\hline 140 & 109 & 106.5 & 108.8 \\
\hline 150 & 120 & 118 & 120.5 \\
\hline
\end{tabular}

\section{Conclusion}

In this paper, a methodology to design $8 \mathrm{MW}$ (peak), $30 \mathrm{~kW}$ (average) klystron gun is presented. Electron gun designed using Vaughan synthesis and optimized with Trak code. The optimized design of the electron gun is validated with CST Particle Studio and Opera-3D codes and VI characteristics of the electron gun are found in close agreement with all three codes.

\section{Acknowledgement}

The authors are thankful to all colleagues of 'vacuum electron device area' and Director CSIR-CEERI for their support and encouragement. 


\section{References}

[1] R.Zhang and Y. Wang, Six-beam gun design for a high power multiple-beam klystron, in IEEE Transactions on Electron Devices, vol. 6o, no. 7, pp. 2395-2401, 2013.

[2] Deepender Kant, L M Joshi, V Janyani, Design of a multi beam electron gun for $352.2 \mathrm{MHz}, 100 \mathrm{~kW}(\mathrm{CW})$ power klystron, Journal of Electromagnetic Waves and Application, vol. 32, no. 3, pp. 306-318, 2018.

[3] Tara Mchugh, Microwave processing heats up, Food Technology Magzine, vol. 70, no. 10, 2016.

[4] J. R. M. Vaughan, Synthesis of the Pierce gun, IEEE Transaction on Electron Devices, vol. 28, no. 1, pp. 37-41, Jan. 1981, doi: 10.1109/TED.1981.20279.

[5] Trak code user Manual.

[6] CST Design Studio, 2018, user manual.

[7] Opera-3d user guide.

[8] A. S. Gilmour Jr., Microwave Tubes. 1st ed., Artech House, 1986.

[9] M. J. Smith and G. Phillips, Power Klystron Today. 1st, ed., John Wiley \& Sons Inc, 1995.
[10] Robert J. Barker, Neville C. Luhmann, John H. Booske, Gregory S. Nusinovich, Modern Microwave and Millimeterwave Power Electronics. Wiley IEEE Press, 2005

\section{Biography of the authors}

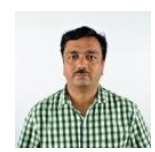

Deepender Kant received B.E. (Hons.) degree in Electrconics and Communication Engineering from University of Rajasthan, India in 2002 and M.Tech. (Hons.) in Electronics Engineering (with specilalization in Microwaves) from IIT-BHU, Varanasi in 2011. Currently he is working as Principal Scientist at CSIR-CEERI, Pilani, India. His research area includes design and development of high power microwave devices and their applications in various systems.

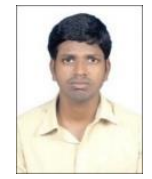

Atmakuru Nagaraju received B.Tech degree in Electrconics and Communication Engineering from KSRMCE kadapa, India in 2010 and M.Tech in RF \& Microwave engineering from IIT Kharagpur in 2013. Currently he is Scientist in Micrwave Devices Area at CSIR-CEERI, Pilani-333031, India. His research areas are Antennas, millimetre technologies, electron gun and magnetic focusing system for microwave tubes. 